Supporting Information.

\title{
Achieving High-Quality Sn-Pb Perovskite Films on Complementary Metal-Oxide-Semiconductor- Compatible Metal/Silicon Substrates for Efficient Imaging Array
}

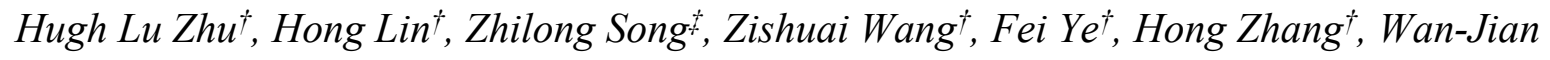
Yin ${ }^{+}$Yanfa Yans, Wallace C.H. Choy ${ }^{*,+}$

$\dagger$ Department of Electrical and Electronic Engineering, The University of Hong Kong, Hong Kong 999077, SAR China

$\ddagger$ Soochow Institute for Energy and Materials InnovationS (SIEMIS), College of Physics, Optoelectronics and Energy \& Collaborative Innovation Center of Suzhou Nano Science and Technology, Soochow University, Suzhou 215006, China

$\S$ Department of Physics and Astronomy and Wright Center for Photovoltaics Innovation and Commercialization, The University of Toledo, 2801 W. Bancroft Street, Toledo, OH 43606, USA

* E-mail: chchoy@eee.hku.hk (Wallace C.H. Choy). 

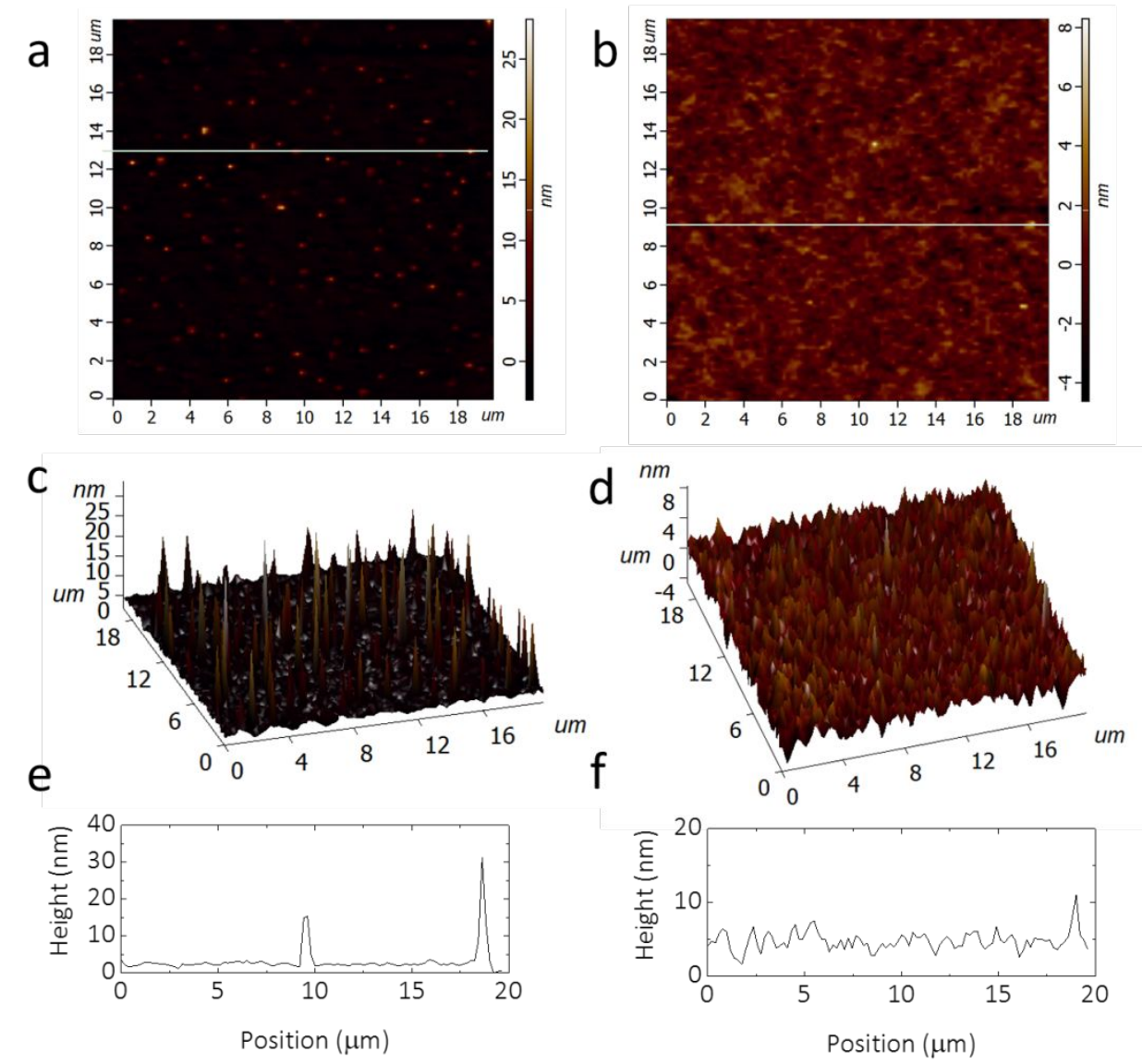

Figure S1. (a, b) Top-view AFM images, (c, d) corresponding three-dimension-view AFM images, and (e, f) cross-sectional surface height profiles of $\mathrm{Cu} / 3$ mercaptopropyltrimethoxysilane/silicon substrates and PEDOT:PSS $/ \mathrm{MoO}_{3} / \mathrm{Cu} / 3-$ mercaptopropyltrimethoxysilane/silicon substrates, respectively. 
Note $\mathrm{S} 1$ Discussion on functions of the $\mathrm{MoO}_{3} / \mathrm{PEDOT}$ :PSS bilayer

It is found that thermal-evaporated metal films with vertical needle-type islands show the height of around $32.2 \mathrm{~nm}$, as shown in Figure S1c. Such sharp metal islands would penetrate perovskites and their interconnections would induce severe recombination and chemical reactions in-between. Hence, an effective intermediate layer between metal electrodes and perovskites should be introduced to smooth the underlay of perovskite films as well as serves as the carrier extraction layer.

In our work, a thin $\mathrm{MoO}_{3}$ layer $(\sim 10 \mathrm{~nm})$ was firstly thermally deposited atop metal films. It works as the hole extraction layer and provides hydrophilic surfaces to the sequential layer.

The aqueous based PEDOT:PSS can therefore form a smooth and well-covered film with a thickness of $\sim 50 \mathrm{~nm}$ on the surface of $\mathrm{MoO}_{3}$. Notably, apart from the hole extraction ability, aqueous solution-processed PEDOT:PSS harbors excellent wetting and soft amorphous properties, which not only provides hydrophilic surfaces to perovskites, but also effectively smooths its underlay (i.e. $\mathrm{MoO}_{3} /$ metal films), as confirmed in Figure S1d. Consequently, after deposition of PEDOT:PSS/ $\mathrm{MoO}_{3}$, the underlay of perovskite films is considerably smoothed, facilitating the growth of high-quality perovskite films (see the figure below).

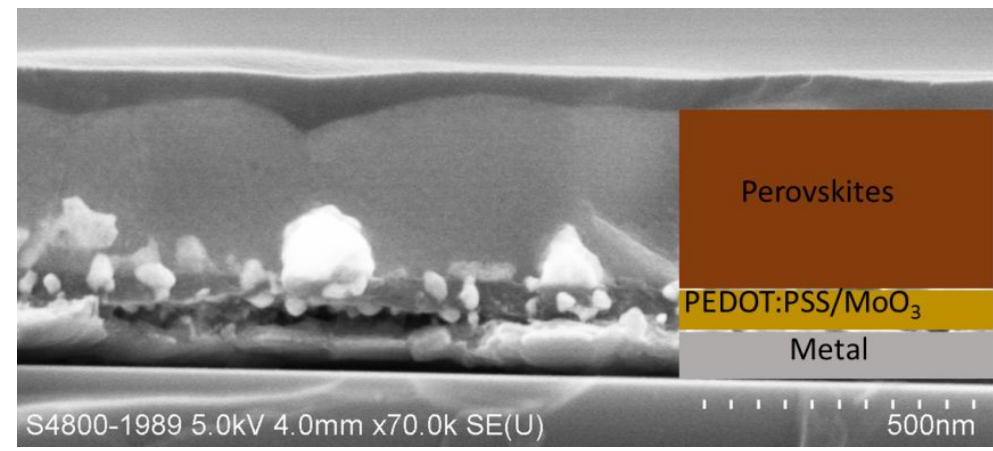




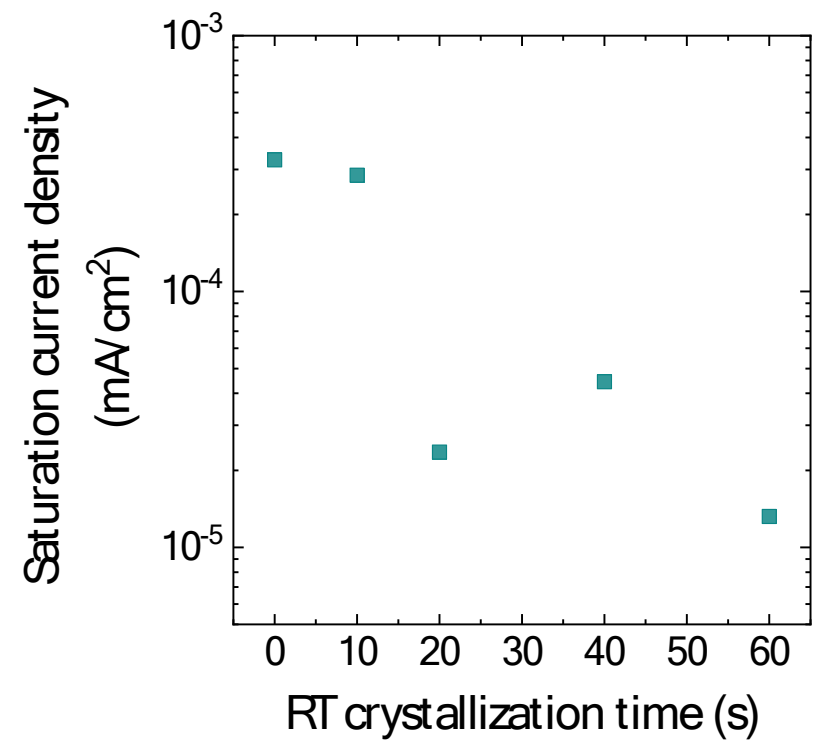

Figure S2. The saturation current density of PVSK-PDs as a function of room-temperature crystallization time. The saturation current density is extracted from the dark current-voltage measurement of PVSK-PDs. 
Note S2 The optimization of top transparent metal electrodes.

It should be noted that in selection of the top transparent electrode, as compared to other metals such as $\mathrm{Cu}$ and $\mathrm{Ag}$, Au with the same thickness offer highest transmittance in the wavelength range covering visible to near-infrared regions (Figure Note S1), low sheet resistance of rough $10 \mathrm{ohm} / \square$ and best stability (Figure Note S2). Hence, thin Au films has been selected as the conductive and transparent window. It should be noted that thin $\mathrm{Cu}$ films showed rapidly increased sheet resistance, indicating inferior stability as shown in Figure Note S2, which is different from the stable thick $\mathrm{Cu}$ electrodes typically formed on perovskite devices. Finally, a thick MoO3 capping layer is further employed to reduce the reflectance loss of thin Au electrodes (Figure Note S3).

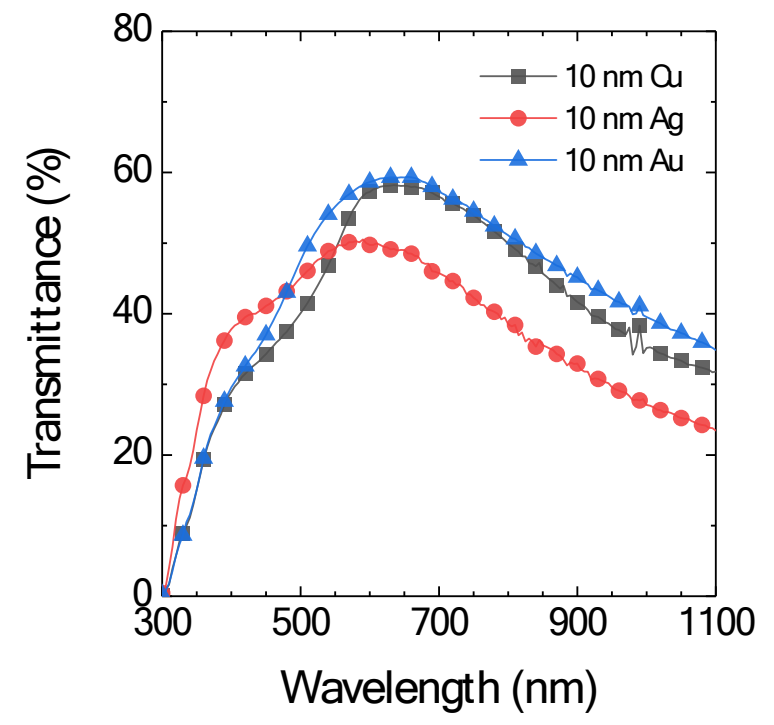

Figure Note S1. Transmittance spectra of thin metal films $(\mathrm{Cu}, \mathrm{Ag}, \mathrm{Au})$ deposited on ZrAcac/PCBM/glass substrates. 

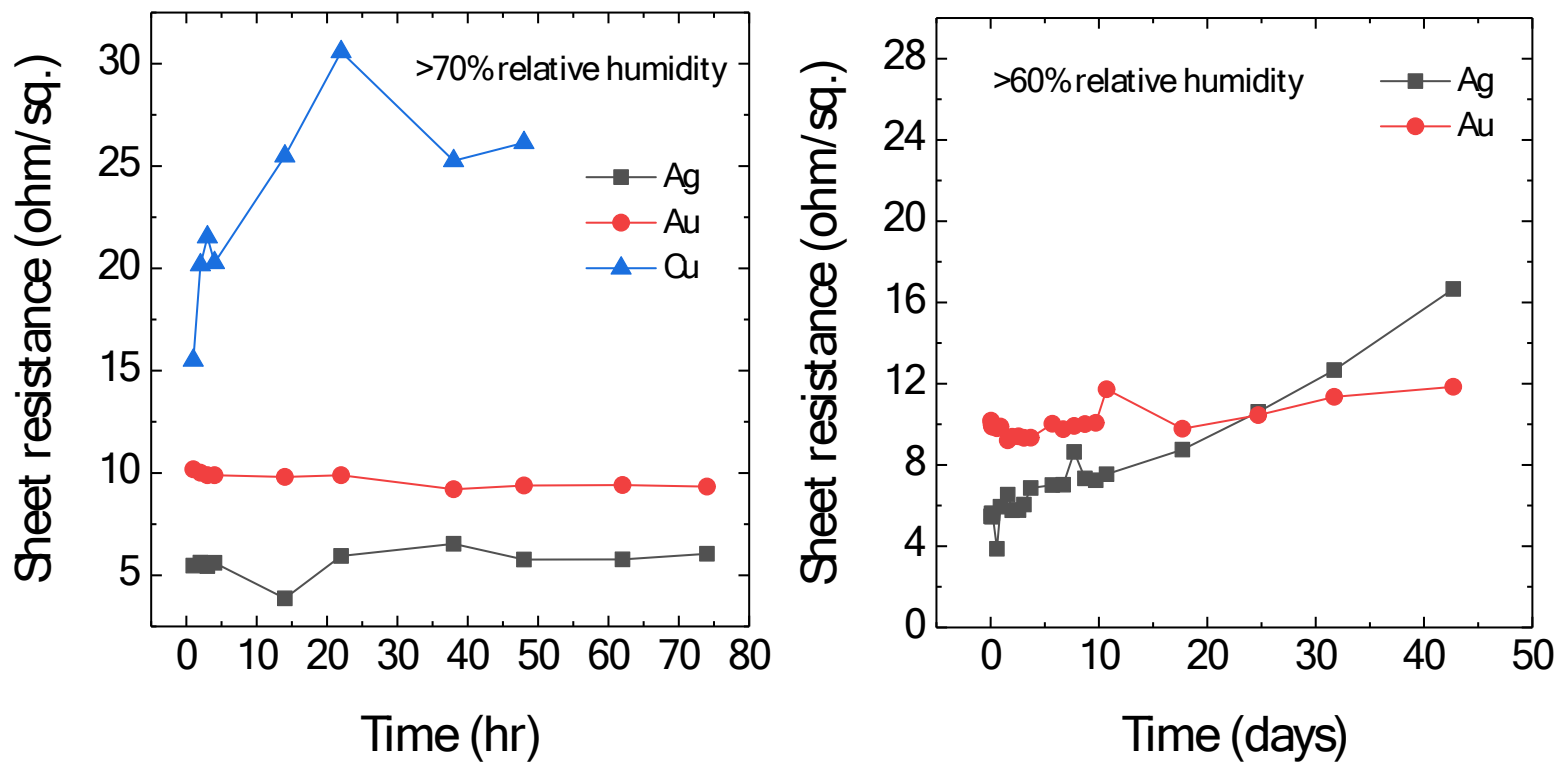

Figure Note S2. (Left) Sheet resistance evolution of 10-nm-thickness $\mathrm{Ag}, \mathrm{Au}$ and $\mathrm{Cu}$ transparent films on ZrAcac/PCBM/glass substrates. (Right) Long-term stability of 10-nmthickness Ag and Au transparent films on ZrAcac/PCBM/glass substrates.

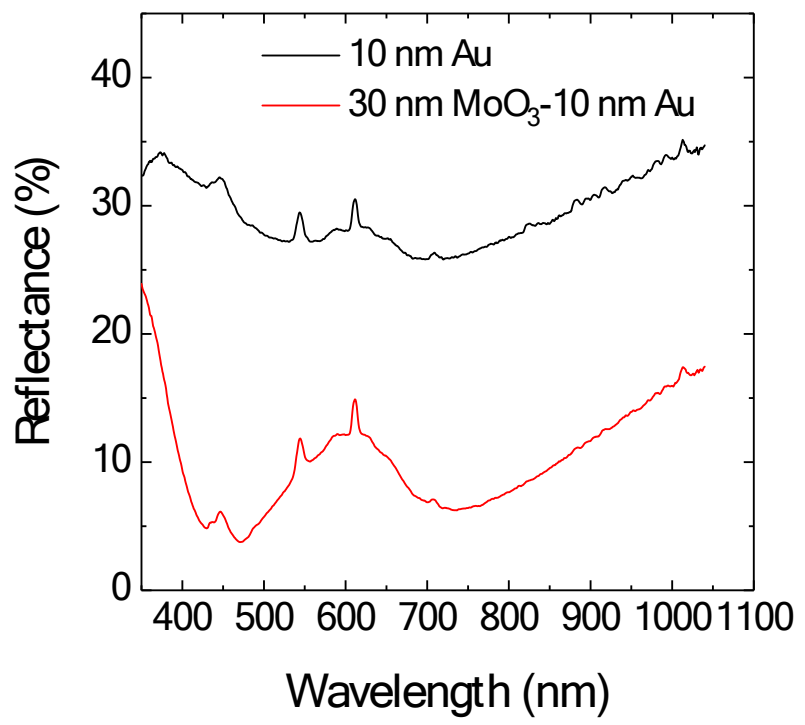

Figure Note S3. Reflectance spectra of thin Au films with or without a $\mathrm{MoO}_{3}$ capping layer. 

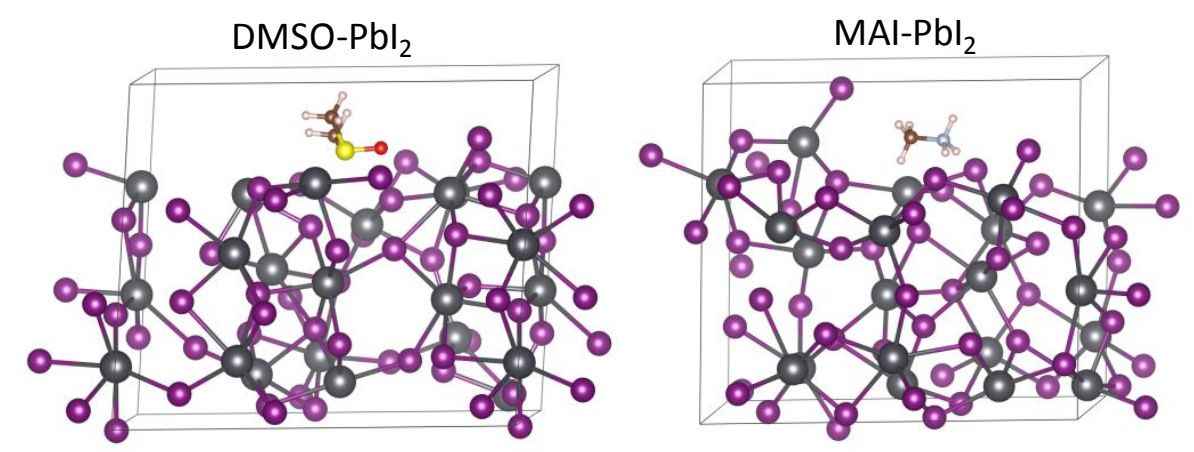

Figure S3. Schematics of $\mathrm{PbI}_{2}-\mathrm{DMSO}$ and $\mathrm{PbI}_{2}-\mathrm{MAI}$. The calculated value of dissociation energy are summarized in the table below.

\begin{tabular}{|l|l|l|}
\hline & $\mathrm{SnI}_{2}$ & $\mathrm{PbI}_{2}$ \\
\hline Surface energy (eV) & -123.434 & -165.281 \\
\hline Dissociation energy for DMSO bonding (eV) & 0.550 & 0.109 \\
\hline Dissociation energy for MAI bonding (eV) & 0.676 & 0.612 \\
\hline
\end{tabular}




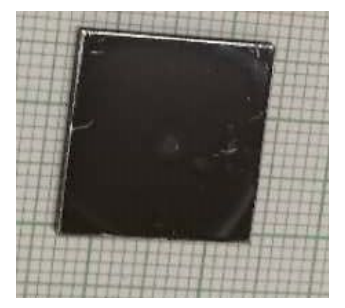

Figure S4. The image of Sn-rich binary perovskite film on glass substrates. After anti-solvent washing, Sn-rich binary perovskite precursor film spontaneously turned from light brown into dark color. 

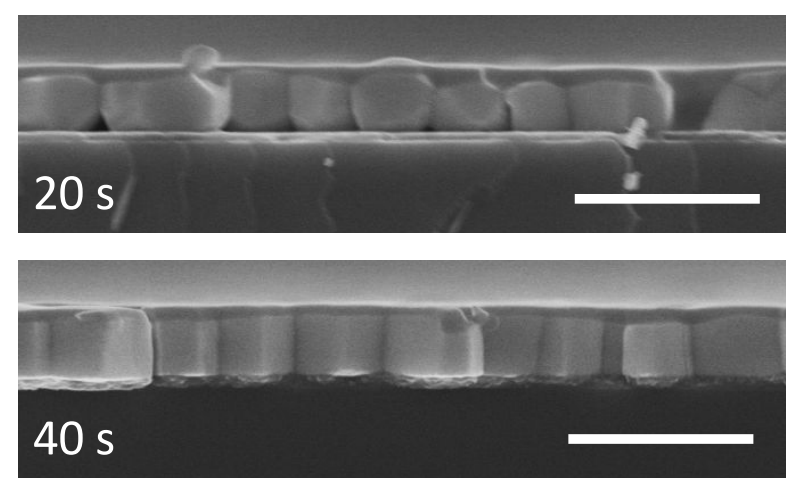

Figure S5. Cross-sectional SEM images of perovskite films with the various time of roomtemperature crystallization: (top) $20 \mathrm{~s}$ and (bottom) $40 \mathrm{~s}$. After room-temperature crystallization, these precursor films were treated by thermal annealing to produce resultant films. 


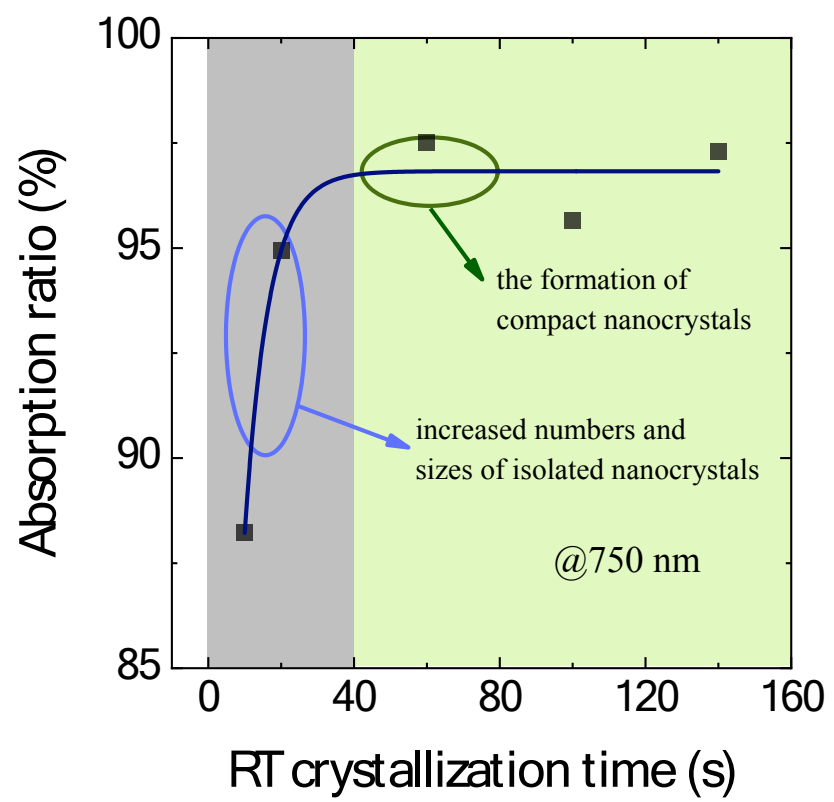

Figure S6. Absorption ratio against room-temperature crystallization time at $750-\mathrm{nm}$ wavelength. The absorption ratio is determined by the absorption intensity of precursor films over that of corresponding post-annealed films. The black line is a guide to the eye. 

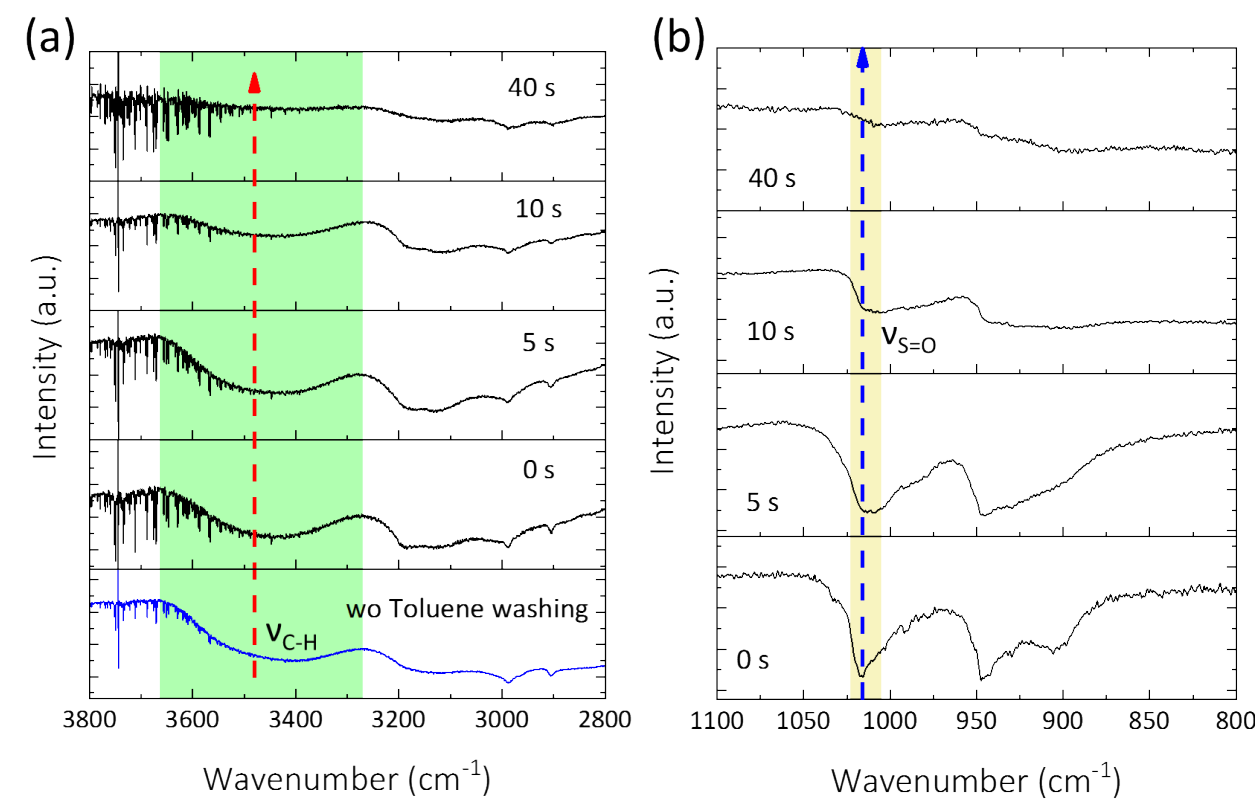

Figure S7. (a) FTIR spectra at $2800-3800 \mathrm{~cm}^{-1}$ of Sn-rich binary perovskite precursor films before and after the anti-solvent washing. The the $\mathrm{C}-\mathrm{H}$ stretching vibration from solvents (3300-3500 $\left.\mathrm{cm}^{-1}\right)$ reduces with the increased crystallization time, indicating the removal of solvents. (b) FTIR spectra at $800-1100 \mathrm{~cm}^{-1}$ of perovskite precursor films at different RT crystallization time. The $\mathrm{S}=\mathrm{O}$ stretching vibration $\left(v_{\mathrm{S}=\mathrm{O}}\right)$ of DMSO molecules $\left(1018-1007 \mathrm{~cm}^{-}\right.$ $\left.{ }^{1}\right)$ originates from DMSO-based intermediates. 


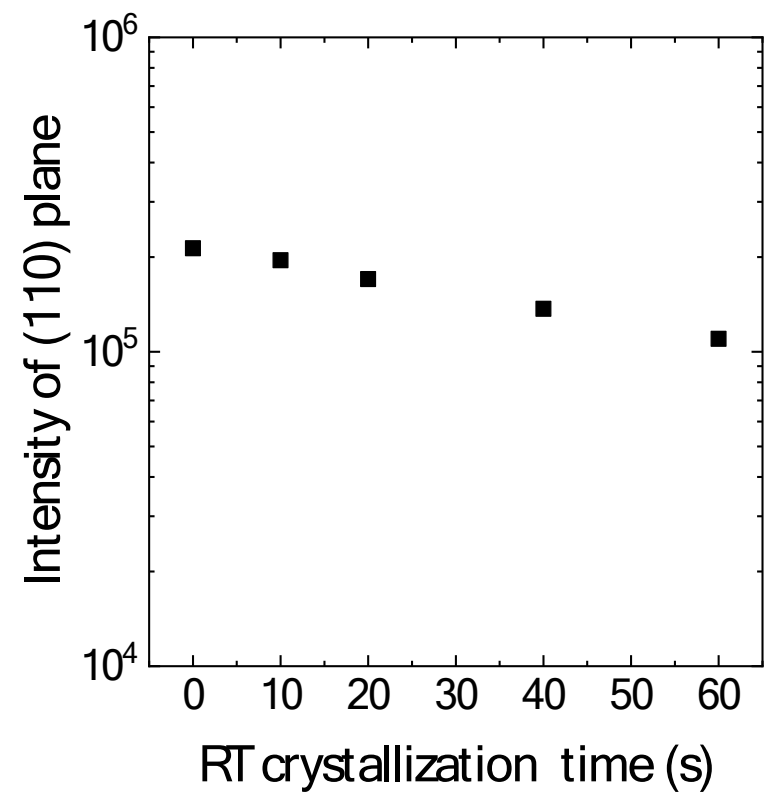

Figure S8. Intensity of (110) planes of Sn-rich binary perovskites as a function of the roomtemperature crystallization time. 

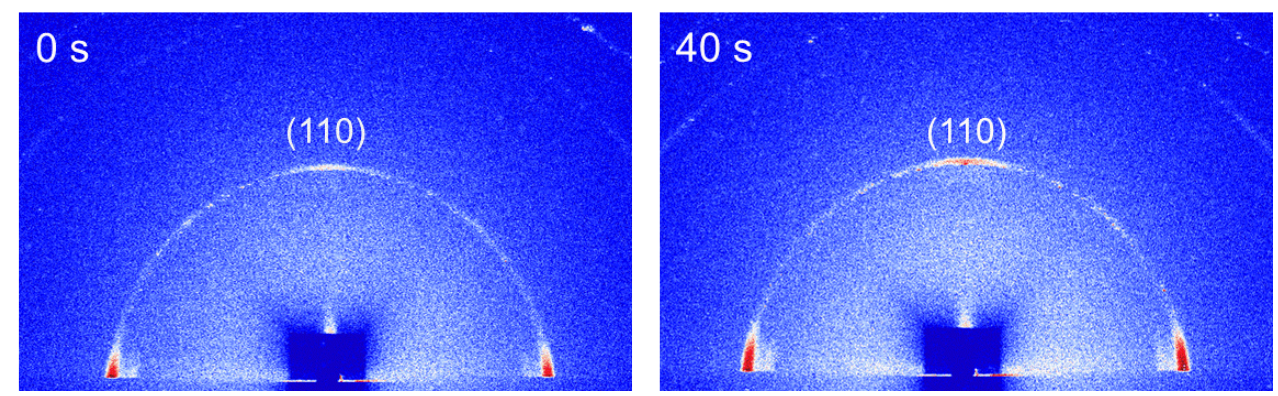

Figure S9. GIWAXS patterns of Sn-rich binary perovskites with the room-temperature crystallization time of $0 \mathrm{~s}$ and $40 \mathrm{~s}$, respectively. 

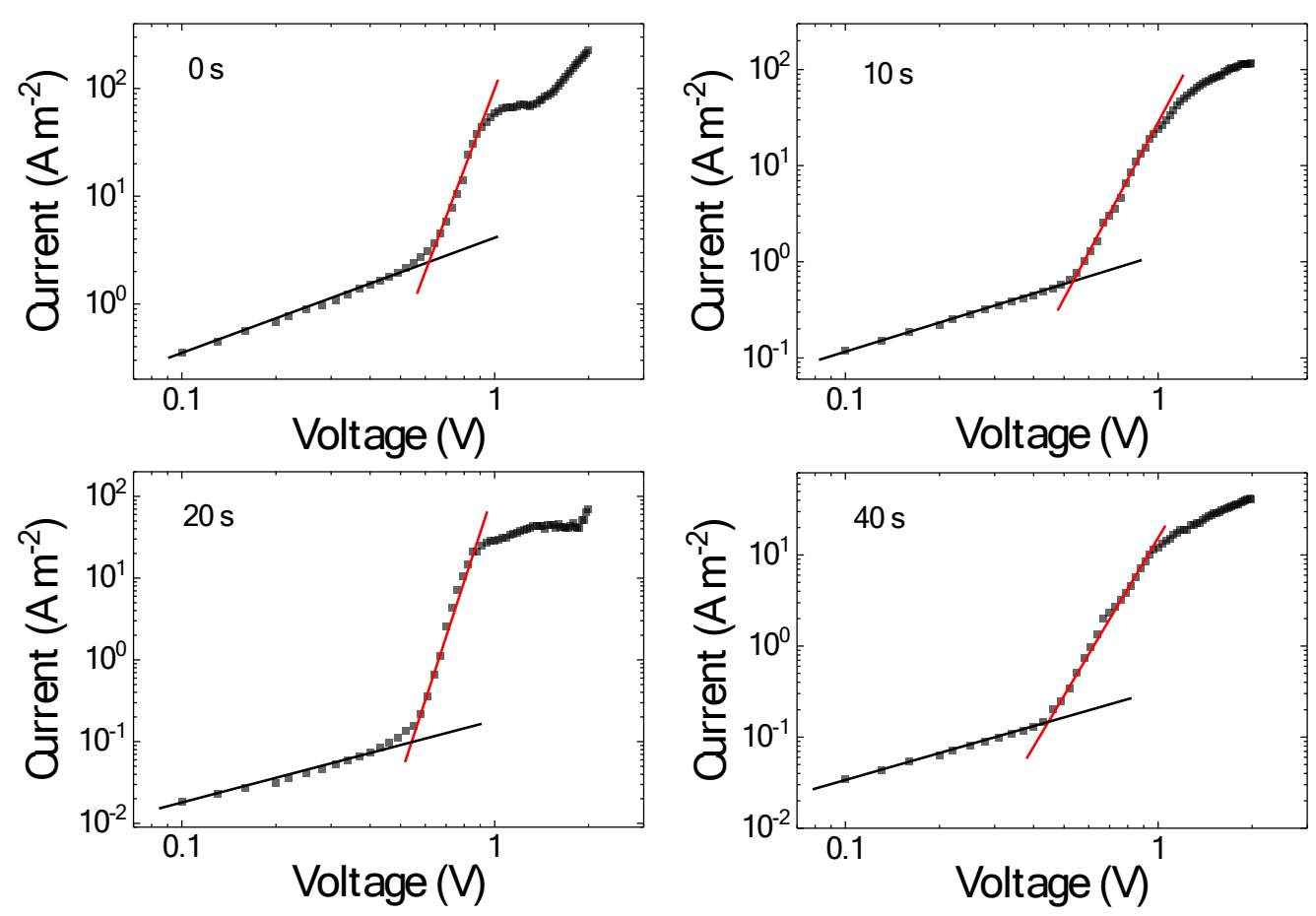

Figure S10. Current-voltage curves of hole-only devices employing Sn-rich perovskites processed by various room-temperature crystallization time. Fitting based on the linear ohmic regime and the trap-filled regime determine the trap-filled limit voltages for trap density estimation. 


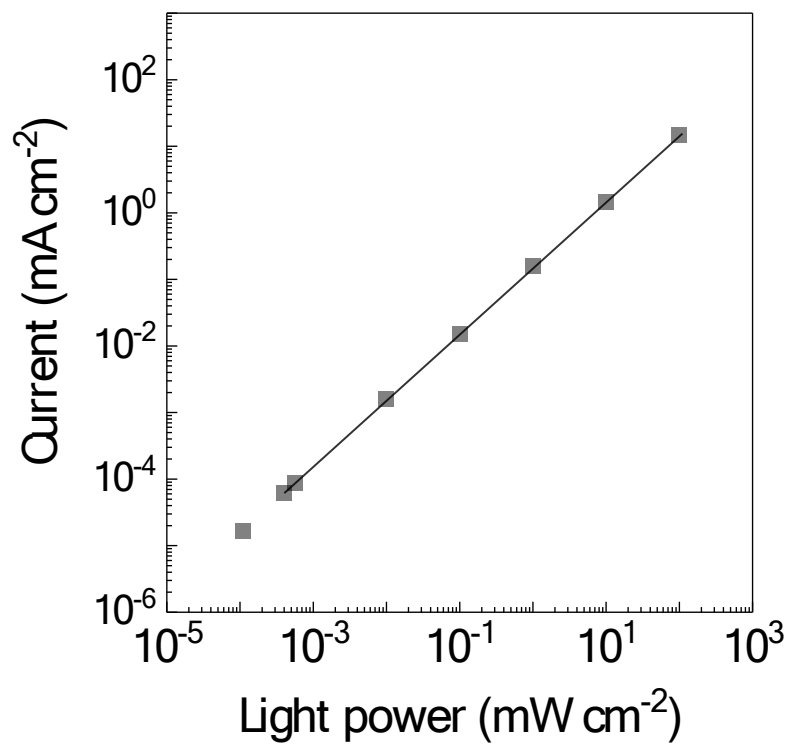

Figure S11. Photocurrent density dependent of the light power. Dynamic range of a imaging sensor is calculated by $20 \log \left(P C_{\max } / P C_{\min }\right)$, where $P C_{\max }$ is the maximum linearly detectable light power, $P C_{\min }$ is the minimum linearly detectable light power. According to the definition of dynamic range, the dynamic range value of Sn-rich binary PVK-PDs is estimated to be 100 $\mathrm{dB}$. 


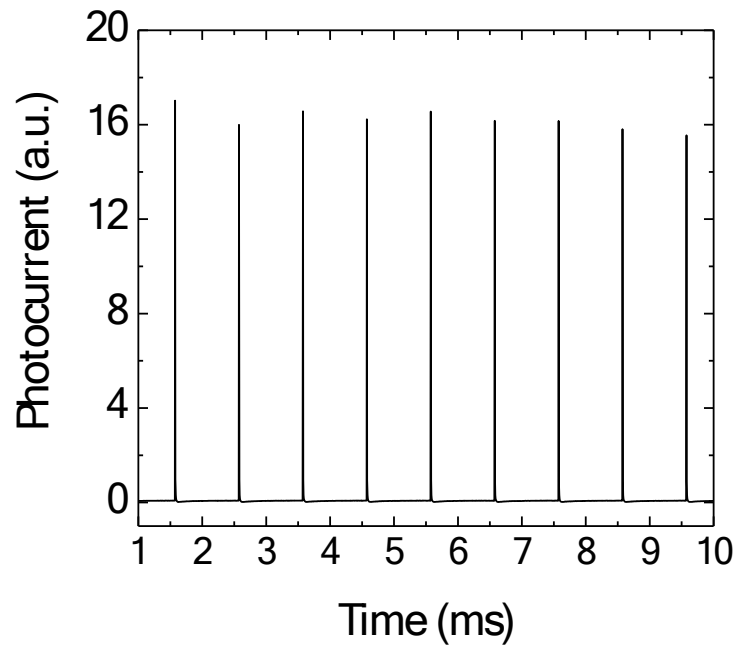

Figure S12. Repeatable photocurrent temporal response of Sn-rich binary PVK-PDs constructed on $\mathrm{Cu} /$ silicon substrates. 


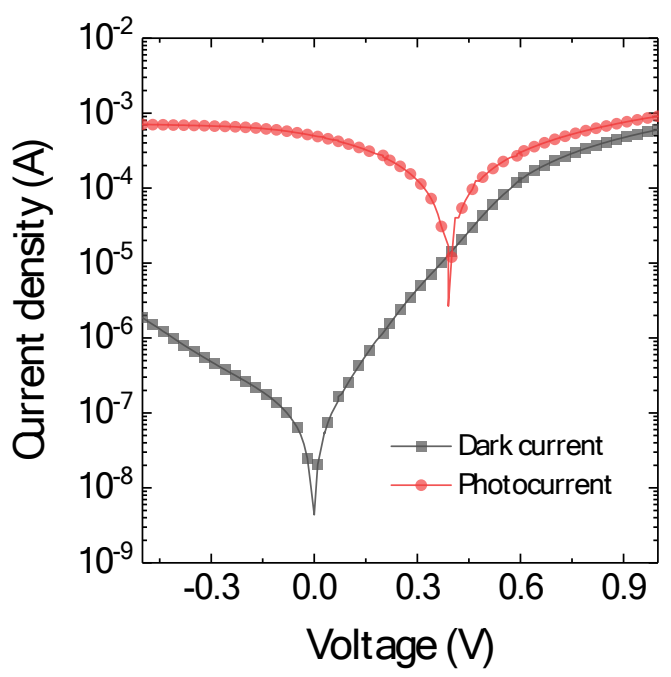

Figure S13. Current density as a function of voltage of Sn-rich binary PVK-PDs in a dark room and under light illumination, respectively. 


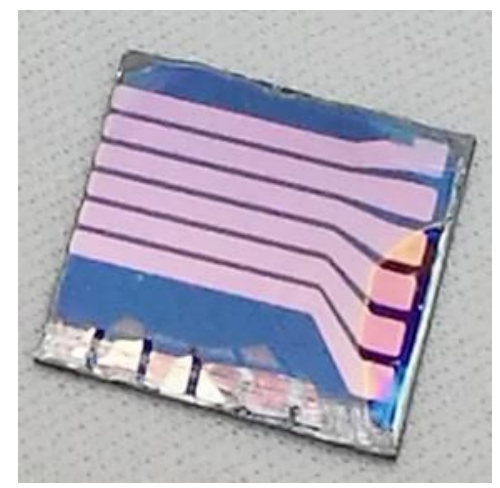

Figure S14. The image of our developed metal/silicon-substrate-based Sn-rich binary PVKPDs array of $6 \times 6$ pixels. 